\title{
Biochemical and histopathologic assessment of effects of acitretin on epiphyseal growth plate in rats
}

\author{
Sevda Onder ${ }^{1}$, Serap Gunes Bilgili², Gulay Bulut ${ }^{3}$, Huseyin Tugrul Celik ${ }^{4}$, Serpil Oguztuzun ${ }^{5}$, Hacı Onder $^{6}$, \\ Omer Calka², Ayse Serap Karadag?
}

${ }^{1}$ Department of Dermatology, Faculty of Medicine, Ordu University, Ordu, Turkey ${ }^{2}$ Department of Dermatology, Faculty of Medicine, Yuzuncu Yıl University, Van, Turkey ${ }^{3}$ Department of Pathology, Faculty of Medicine, Yuzuncu Yıl University, Van, Turkey ${ }^{4}$ Department of Biochemistry, Faculty of Medicine, Turgut Ozal University, Ankara, Turkey ${ }^{5}$ Department of Biology, Faculty of Arts and Sciences, Kırıkkale University, Kırıkkale, Turkey ${ }^{6}$ Department of Orthopaedics and Traumatology, Ordu University Training and Research Hospital, Ordu, Turkey ${ }^{7}$ Department of Dermatology, Faculty of Medicine, Istanbul Medeniyet University, Istanbul, Turkey

Adv Dermatol Allergol 2020; XXXVII (3): 346-352 DOI: https://doi.org/10.5114/ada.2020.95983

\begin{abstract}
Introduction: Acitretin is a commonly used retinoid in dermatology. Although there are generally known side effects, the effects on the epiphyseal plaque and bone metabolism are not clear in the literature.

Aim: To histopathologically investigate the effects on the epiphyseal plate and assess variations in bone metabolism caused by acitretin.

Material and methods: Three groups were formed with 10 rats in each group. The $1^{\text {st }}$ group $(n=10,5$ male, 5 female) were administered $10 \mathrm{mg} / \mathrm{kg} /$ day oral acitretin solution and the $2^{\text {nd }}$ group $(n=10,5$ male, 5 female) were administered $3 \mathrm{mg} / \mathrm{kg} / \mathrm{day}$ oral acitretin solution. The control group were given normal standard feed and water. Rats were sacrificed at the end of 4 weeks. The proximal tibias were excised and histopathologically and immunohistochemically assessed. Biochemical assessment was also carried out.

Results: Staining with haematoxylin-eosin found reductions in the epiphyseal plate in the $1^{\text {st }}$ and $2^{\text {nd }}$ group compared to the control group, though this situation was not statistically significant. Immunohistochemical studies did not encounter Type II collagen in the epiphyseal bone, proliferative zone and hypertrophic zone in the control group, low dose acitretin solution group and high dose acitretin solution group. Type II collagen was not observed in osteoids and osteoblasts. Type I collagen was not observed in the hypertrophic zone and proliferative zone of any group. Conclusions: Our data show that though acitretin caused degeneration of the epiphyseal plate, it did not cause clear thinning and we identified no significant variations in bone metabolism markers.
\end{abstract}

Key words: acitretin, epiphyseal plate, bone metabolism, rat.

\section{Introduction}

Retinoids are defined as both natural and synthetic compounds with the functional characteristics of vitamin A, affecting cellular growth and differentiation, immunomodulation, tumour promotion, and malignant potential of cells [1].

Though the majority are well tolerated, retinoids are reported to have many side effects. The most common side effects are teratogenicity, mucocutaneous effects, elevated transaminase levels and hyperlipidaemia [1, 2].

With the use of retinoids there are various effects on the musculoskeletal system. The most commonly observed effects are arthralgia, myalgia, hyperostosis, reduced bone mineralization, and calcification of ligaments and tendons. Rarely tendinopathies, periosteal proliferations and premature epiphyseal closure are reported [2, 3].

Acitretin is a well-known retinoid derivative medication used for treatment of psoriasis patients. However, its use is frequently limited due to side effects, especially for long-term treatment. Though skeletal side effects are associated with hypervitaminosis A, the association with retinoid treatment is controversial in the literature. As a result of long-term retinoid treatment, diffuse idiopathic skeletal hyperostosis (DISH) syndrome, osteoporosis,

Address for correspondence: Sevda Önder, Department of Dermatology, Faculty of Medicine, Ordu University, Ordu, Turkey, phone: +90 5306035398, fax: +90 452 2265228, e-mail: drsevdaonder@gmail.com Received: 1.09.2018, accepted: 23.10.2018. 
calcification of ligaments and premature epiphyseal closure are reported. However, due to the lack of prospective controlled studies, no causative relationship could be determined [4].

Vitamin A hypervitaminosis is a known cause of premature epiphysiodesis. This effect is a side effect that may be caused by retinoid derivative medications. In the literature, different bone and epiphyseal side effects are reported to develop with different retinoids. However, it is impossible to say whether all retinoids carry the same risk without epidemiological studies [2].

To the best of our knowledge, there is no immunohistochemical study investigating the effects of the retinoid vitamin A derivative of acitretin on the epiphyseal growth plate.

\section{Aim}

In this study, we aimed to investigate the effects of high- and low-dose acitretin on the epiphyseal growth plate and bone markers in a rat model.

\section{Material and methods}

The study received permission from the Yüzüncü Yıl University Animal Experiments Local Ethics Committee Chair and was supported by the Yüzüncü Yıl University Scientific Research Project Chair. The study used 15 male and 15 female Wistar Albino rats, weighing 250-300 g. The rats were $4-8$ weeks old and kept at room temperature in cages containing five animals. The experimental animals were given an appropriate diet and the room had 12-hour light-dark cycles. Three groups were created with 5 male and 5 female rats in each group. The $1^{\text {st }}$ group was given $10 \mathrm{mg} / \mathrm{kg} /$ day dose (high dose) of acitretin solution. The $2^{\text {nd }}$ group were given $3 \mathrm{mg} / \mathrm{kg} /$ day (low dose) acitretin solution. The $3^{\text {rd }}$ group was only given standard feed and water. The acitretin solution (Neotigason capsule: Roche/Greek) was diluted to the appropriate dose with deionized water and was administered orally for 4 weeks to the rats in group 1 and group 2 . On the $29^{\text {th }}$ day of the study, all rats were administered ketamine- $\mathrm{HCl}$ (hydrochloride) (100 mg/kg; Ketanest 100 mg/ml) intraperitoneally, and thus anaesthesia was ensured for blood sampling and sacrifice of rats. Serum calcium, magnesium, phosphorus, 1,25-OH D vitamin, 25-OH D vitamin, bone alkaline phosphatase, parathormone, and osteocalcin levels were measured. Both tibial bones of all rats were excised. Variations in the epiphyseal plates were assessed histopathologically and immunohistochemically.

\section{Measurement of serum values}

Serum calcium, magnesium, and phosphorus values were photometrically measured using a Roche Diagnostic COBASc501 Japan device (HITACHI High Technologies Corporation, Tokyo, Japan). Rat 1,25-OH D vitamin was studied with a CSB-E13342r Cusabio ELISA kit. Rat
25-OH D vitamin was studied with a CSB-E08098r Cusabio ELISA kit. Rat BALP was studied with a CSB-E11865r Cusabio ELISA kit. Rat PTH was studied with a CSB-E07866r Cusabio ELISA kit. Rat osteocalcin was studied with a CSB-E05129r Cusabio ELISA kit.

\section{Histopathologic investigation of epiphyseal plate}

Rat tibias fixated in $10 \%$ buffered formaldehyde were decalcified with formic acid. For investigation with a light microscope, an automatic vacuum tissue monitoring device was used. The tissue was submerged in paraffin blocks. Sections were taken with four-micron thickness. These sections placed on slides were stained with haematoxylin-eosin, toluidine blue and Masson trichrome for histopathologic assessment. Assessments measured the resting zone, proliferative and hypertrophic zones of the epiphyseal growth plate. For each bone rudiment, three sections parallel to the long axis of the bone at 30-micron intervals were taken. Then the mean value for the three sections was calculated.

Cartilage was stained with collagen type I and type II stains. Proximal tibias were assessed immunohistochemically using Polyclonal AntiCOLIA1 Antibody, PA2140-2 catalogue number (Boster Biological Technology, USA) and Collagen II Antibody C0155-1, C0155-2, C0155 catalogue number (Boster Biological Technology, USA) against type I and type II collagen. Assessment of collagen staining was made according to intensity of staining as no stain (-) (score 0), suspected staining $(+) /(-)$ (score 1$)$, weak staining $(+)$ (score 2$)$, moderate staining (++) (score 3 ) and strong staining (+++) (score 4). According to the distribution of staining, assessments were as follows: staining of 0-10\% of cells (+), staining of $11-50 \%$ of cells $(++)$, staining of $51-75 \%$ of cells $(+++)$ and staining of $76-100 \%$ of cells $(++++)$.

\section{Statistical analysis}

The measurements were statistically analysed. Descriptive statistics for continuous variables include mean, standard deviation, minimum and maximum, while categorical variables are given as a number and percentage. For comparison of group means for continuous variables, the one-way analysis of variance was used. The Duncan test was used to determine differing groups after variance analysis. The $\chi^{2}$ test was used to determine the correlation between categorical variables. The statistical significance level was taken as 5\% for calculations and calculations were completed with the SPSS statistical program.

\section{Results}

\section{Histopathologic assessment findings}

In our study, each group contained 5 male and 5 female rats. Data were assessed both for the effect of both sexes and independent of sex. Assessments measured the resting zone, proliferative and hypertrophic zones of the epiphyseal growth plate. The resting zone mean 
Table 1. Comparison of the histopathological examination of the control group with low-dose and high-dose acitretin solution groups

\begin{tabular}{|c|c|c|c|c|c|c|c|}
\hline Parameter & & $N$ & Med. & St. dev. & Min. & Max. & $P$-value \\
\hline \multirow{3}{*}{$\begin{array}{l}\text { Resting zone } \\
{[\mu \mathrm{m}]}\end{array}$} & Control & 10 & 53.75 & 13.65497 & 25.00 & 75.00 & \multirow[t]{3}{*}{0.279} \\
\hline & $2^{\text {nd }}$ group & 10 & 58.25 & 9.57790 & 47.50 & 80.00 & \\
\hline & $1^{\text {st }}$ group & 10 & 62.00 & 10.19259 & 50.00 & 75.00 & \\
\hline \multirow{3}{*}{$\begin{array}{l}\text { Proliferative zone } \\
{[\mu \mathrm{m}]}\end{array}$} & Control & 10 & 108.75 & 31.38582 & 50.00 & 142.50 & \multirow[t]{3}{*}{0.723} \\
\hline & $2^{\text {nd }}$ group & 10 & 116.75 & 21.53969 & 87.50 & 150.00 & \\
\hline & $1^{\text {st }}$ group & 10 & 116.75 & 22.45520 & 75.00 & 150.00 & \\
\hline \multirow{3}{*}{$\begin{array}{l}\text { Hypertrophic zone } \\
{[\mu \mathrm{m}]}\end{array}$} & Control & 10 & 100.75 & 13.89694 & 75.00 & 125.00 & \multirow[t]{3}{*}{0.001} \\
\hline & $2^{\text {nd }}$ group & 10 & 75.00 & 17.67767 & 50.00 & 112.50 & \\
\hline & $1^{\text {st }}$ group & 10 & 67.00 & 16.27882 & 37.50 & 87.50 & \\
\hline \multirow{3}{*}{$\begin{array}{l}\text { Growth plate } \\
{[\mu \mathrm{m}]}\end{array}$} & Control & 10 & 263.25 & 44.78420 & 175.00 & 312.50 & \multirow[t]{3}{*}{0.454} \\
\hline & $2^{\text {nd }}$ group & 10 & 243.20 & 32.93360 & 194.50 & 312.50 & \\
\hline & $1^{\text {st }}$ group & 10 & 245.75 & 36.13343 & 187.50 & 300.00 & \\
\hline
\end{tabular}

value was $53.75 \mu \mathrm{m}$ in the control group, $58.25 \mu \mathrm{m}$ in the $2^{\text {nd }}$ group and $62 \mu \mathrm{m}$ in the $1^{\text {st }}$ group given a high dose solution. The mean height of the proliferative zone was $108.75 \mu \mathrm{m}$ in the control group, $116.75 \mu \mathrm{m}$ in the $2^{\text {nd }}$ group and $116.75 \mu \mathrm{m}$ in the $1^{\text {st }}$ group. The mean height of the hypertrophic zone was $100.75 \mu \mathrm{m}$ in the control group, $75.00 \mu \mathrm{m}$ in the $2^{\text {nd }}$ group and $67.00 \mu \mathrm{m}$ in the $1^{\text {st }}$ group. The mean height of the whole growth plate was $263.25 \mu \mathrm{m}$ in the control group, $243.20 \mu \mathrm{m}$ in the $2^{\text {nd }}$ group and $245.75 \mu \mathrm{m}$ for the $1^{\text {st }}$ group. When all groups are compared in terms of haematoxylin-eosin staining, there was a significant reduction in the hypertrophic zone in the $1^{\text {st }}$ and $2^{\text {nd }}$ groups compared to the control group. There was no statistically significant difference between the hypertrophic zones in the $1^{\text {st }}$ and $2^{\text {nd }}$ groups. There was no statistically significant reduction observed in the other zones. In the comparison of the 3 groups, though the $1^{\text {st }}$ and $2^{\text {nd }}$ group had thickening of the resting zone compared to the control group, this result was not statistically significant. The whole thickness of the growth plate was reduced in the $1^{\text {st }}$ and $2^{\text {nd }}$ group compared to the control group, but this situation was not statistically significant. The comparison of histopathologic investigation of the study groups is given in Table 1.

When the female and male groups are compared between themselves, there was a statistically significant difference in the resting zone in females between the control group and the $1^{\text {st }}$ group given a high dose solution. The group administered a high dose solution was observed to have thickening of the resting zone compared to the control group. The difference in the proliferative zone was found to be statistically significant in the male rats. Males in the control group were identified to have significant thinning of the proliferative zone compared to females. There were statistically significant reductions in the hypertrophic zone both in males and females of the $1^{\text {st }}$ and $2^{\text {nd }}$ groups compared to the control group. However, there was no significant difference in the hypertrophic zone between the $1^{\text {st }}$ and $2^{\text {nd }}$ groups. When the whole thickness of the growth plate is calculated, there was a reduction in the male and female groups but this result was not a statistically significant reduction. Staining with Masson trichrome did not produce significant results.

Assessment with toluidine-blue found the columnar arrangement of chondrocytes in the epiphyseal growth plate was $90.9 \%$ in the control group, $9.1 \%$ in the lowdose solution $2^{\text {nd }}$ group and $0 \%$ in the high-dose solution $1^{\text {st }}$ group with a clear reduction and loss. There was no irregular thickening in the control group, while this was present in all of the $1^{\text {st }}$ and $2^{\text {nd }}$ groups. The increase in metachromasia was observed in $91 \%$ of the control group and $9 \%$ of the $2^{\text {nd }}$ group. There was no increase in metachromasia in the $1^{\text {st }}$ group. Those with a reduction in metachromasia were $47.4 \%$ of the $2^{\text {nd }}$ group and $52.6 \%$ of the $1^{\text {st }}$ group and there was no reduction in metachromasia in the control group. Staining with Masson trichrome produced no significant results.

\section{Immunohistochemical assessment findings}

Immunohistochemical studies did not encounter type II collagen in the epiphyseal bone, proliferative zone and hypertrophic zone of the control group, $1^{\text {st }}$ and $2^{\text {nd }}$ groups. Type II collagen was not observed in osteoids and osteoblasts. Type I collagen was not observed in the hypertrophic zone and proliferative zone of any of the groups. In all groups, type I collagen was observed in the epiphyseal bone, osteoids and osteoblasts with distribution of $4(+++)$. There were no significant differences observed between the groups in terms of type I collagen staining distribution and intensity. Rats in the $2^{\text {nd }}$ group with a low dose solution administered had type I collagen staining 
in the epiphyseal bone, osteoids and osteoblasts with $80 \%$ rate $3(51-75 \%), 10 \%$ rate $2(11-50 \%)$ and $10 \%$ rate 4 (76-100\%). In the control group type I collagen staining in the epiphyseal bone, osteoids and osteoblasts was $60 \%$ rate $3(51-75 \%)$ and $40 \%$ rate $4(76-100 \%)$. In terms of intensity of staining, the difference observed between the groups was not significant $(p=0.230)$. Between the sexes, there was no significant difference observed in staining of cartilage and bone tissue. Preparation samples of the experimental groups are seen in Figures 1-6.

\section{Biochemical assessment findings}

When biochemical parameters are examined, comparison of the 3 groups found no statistically significant differences in 1,25 (OH) vitamin D, osteocalcin, bone alkaline phosphatase (ALP), phosphorus and magnesium levels. There was a statistically significant increase in the $25 \mathrm{OH}$ vitamin D, parathorman (PTH) and calcium levels in the $1^{\text {st }}$ group compared to the control group. There was no significant difference observed between the $2^{\text {nd }}$ group and the control group.

Assessment according to sex found no statistically significant differences in $1,25 \mathrm{OH}$ vitamin D, 25-OH vitamin D, osteocalcin, phosphorus, magnesium and ALP values. There was a significant difference in PTH values in both male and female rats. Females had elevated PTH values in the $1^{\text {st }}$ and $2^{\text {nd }}$ groups compared to the control group. For males, there was an increase in the $1^{\text {st }}$ group compared to the control group, but there was no statistically significant difference between the $2^{\text {nd }}$ group and the

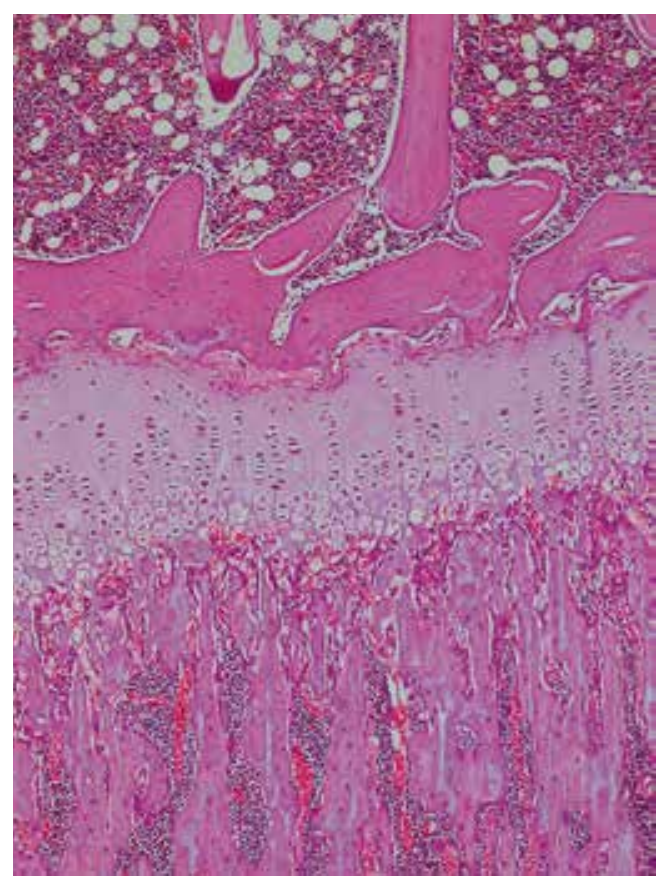

Figure 2. Female rat administered a high-dose acitretin solution. HE 100× bar $=100 \mu \mathrm{m}$ control group. For calcium values, there was a significant increase among females in the $1^{\text {st }}$ group compared to the control group, but this was not present in the $2^{\text {nd }}$ group.

\section{Discussion}

Vitamin A is necessary for metabolic and physiological changes like skin cell differentiation, immune system and gene transcription [5]. Some studies in recent times have shown that retinoic acid receptors are significant factors regulating differentiation and proliferation of chondrocytes $[6,7]$.

The epiphyseal plate, also known as the physis, is a known mammalian growth plate with a highly developed mesoderm-derived cartilage structure. It develops from the osteogenic bud and is responsible for lengthening of the bone. Plates are formed by many cells that divide and mature rapidly. After puberty the cell division of epiphyseal cartilage reduces, bone fully replaces cartilage and epiphyseal plates unite with primary and secondary ossification centres [8].

The growth plate is found between the epiphysis and metaphysis and comprises three regions called the resting zone, proliferative zone and hypertrophic zone. Each

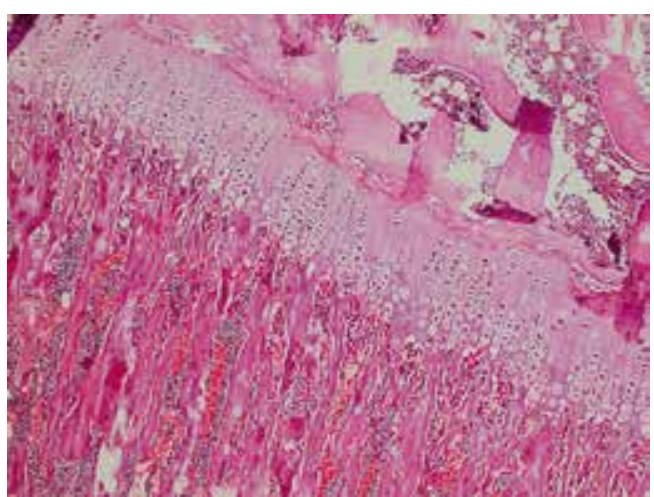

Figure 1. Female rat from the control group. HE 200x, bar $=50 \mu \mathrm{m}$

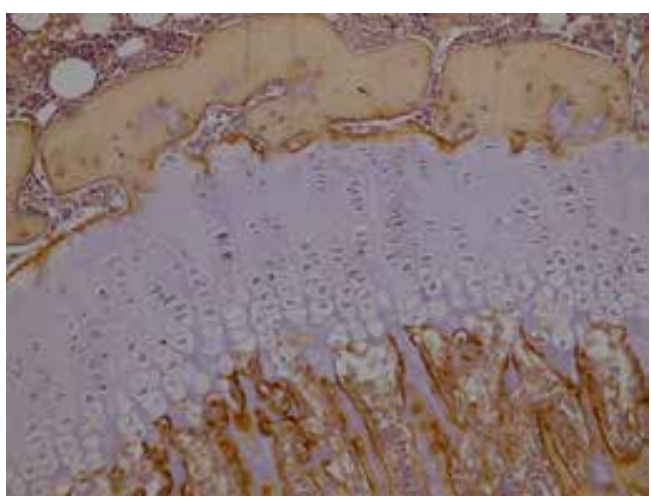

Figure 3. Male rat from the control group. Type I collagen $200 \times$, bar $=50 \mu \mathrm{m}$ 


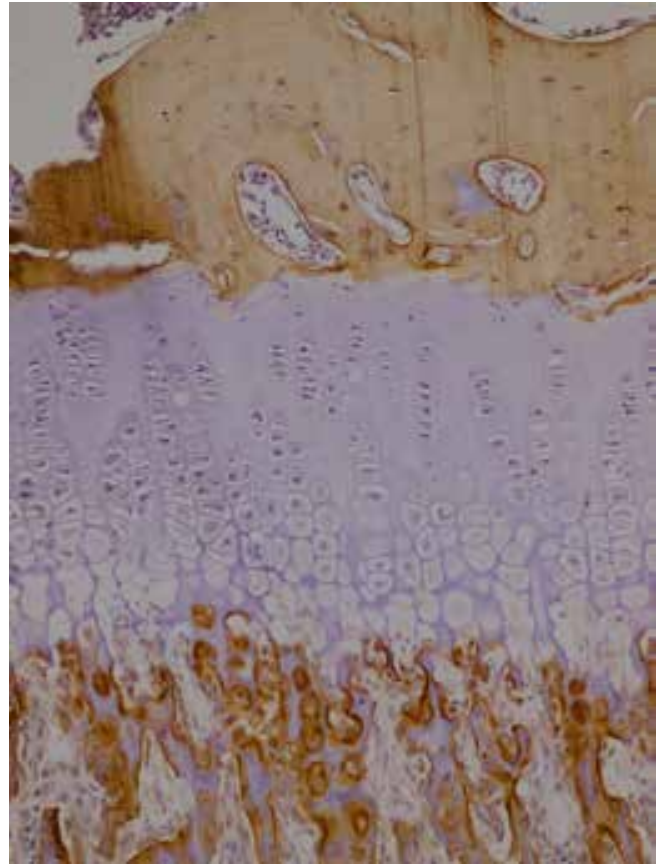

Figure 4. Male rat administered a high-dose acitretin solution. Type I collagen 200x, bar $=50 \mu \mathrm{m}$

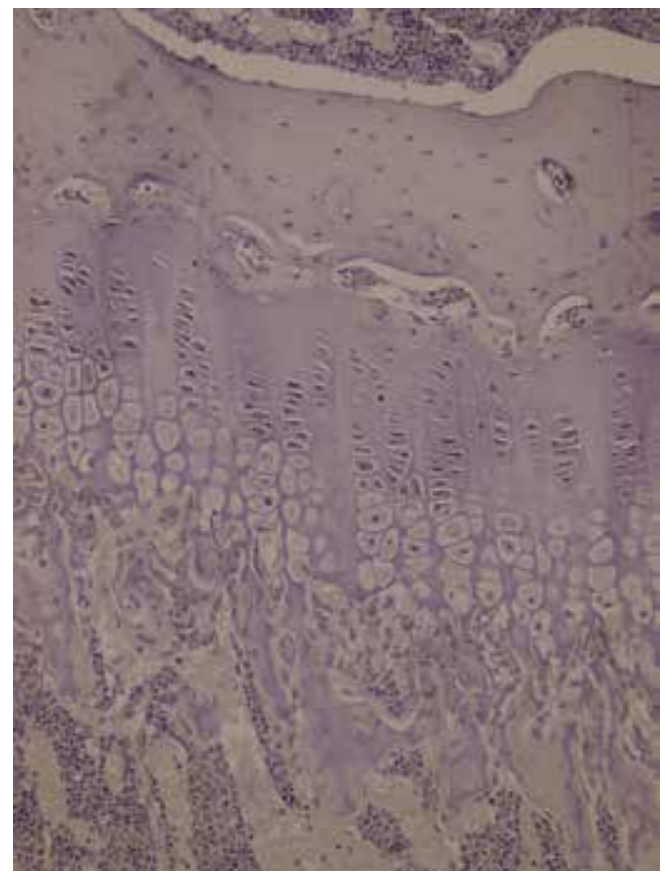

Figure 6. Male rat administered a high-dose acitretin solution. Type II collagen 200x, bar $=50 \mu \mathrm{m}$

min A increases the number and volume of osteoclasts, reducing the osteoid surface causing bone resorption [5].

Kindmark et al. reported reduced type I collagen carboxy-terminal telopeptide levels after 5 days of isotreti-

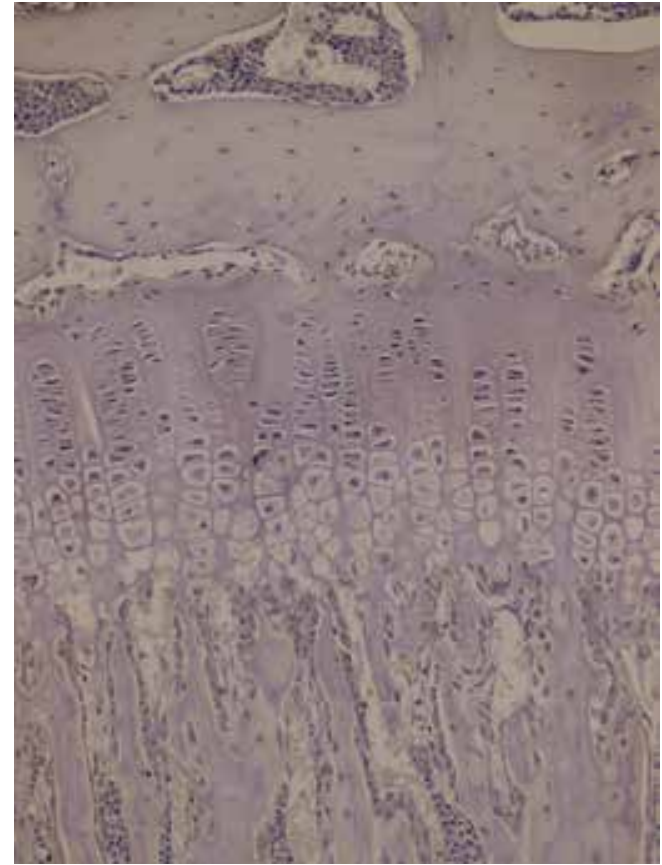

Figure 5. Male rat from the control group. Type II collagen $200 \times$, bar $=50 \mu \mathrm{m}$

region contains different chondrocytes in different stages of differentiation [9]. In the resting zone the extracellular matrix rate is higher than the intracellular rate and cells are resting. This region is characterized by low rates of proliferation and synthesis of proteoglycans and collagen type IIB [8]. In the proliferative zone chondrocytes gain a flattened appearance, division begins and they are organized in columns [10]. In this true germinal layer type II and type XI collagen synthesis increases. Below the proliferative region there are the upper and lower hypertrophic regions and another more distant layer known as the transformation region divided by degeneration [8]. In normal situations, type I collagen is the main component of the bone matrix and the cartilage matrix of the epiphyseal growth plate is not identified [11].

This study identified a significant reduction in the hypertrophic zone without significant reduction in the whole epiphyseal plate thickness in the groups administered acitretin solution compared to the control group. At the same time, though there was no significant reduction in epiphyseal plate thickness, there was significant disruption and deformation of the columnar arrangement of chondrocytes in the groups administered the solution and this arrangement was completely destroyed in the group administered a high dose solution.

Some previous publications have shown that high dose vitamin A use reduces the epiphyseal growth plate [11-13]. Some publications have shown that excess vita- 
noin treatment. Another study showed that retinoids reduced proliferation of chondrocytes in culture, and additionally stopped collagen synthesis in the $48^{\text {th }} \mathrm{h}$ following retinoid exposure [14].

Studies in the literature have shown that retinoids play an important role in skeletal development. Both hypervitaminosis and hypovitaminosis A were shown to disrupt chondrocyte maturation in the growth plates of long bones [7].

Investigations of cartilage in chick embryos to analyse the effect of retinoids on behaviour and development of chondrocytes observed that prehypertrophic and early hypertrophic chondrocytes did not develop maturation under controlled culture conditions, but that maturation occurred when exogenous retinoid was administered [7].

Animal studies have shown that animals treated with vitamin A or derivatives have high rates of chondrocyte loss in epiphyseal cartilage and osteoclast invasion. Some cases have shown premature epiphyseal closure developing with different retinoids [2].

Hyperostosis, tendon and ligament calcification and early epiphyseal closure have been previously reported during use of isotretinoin [15].

Kodaka et al. investigated the epiphyseal growth plate in the proximal tibia of rats administered high-dose vitamin A. This study found that as the dose of vitamin A increases, calcified cartilage formed in the resting zone, calcified areas changed places with bone tissue and local losses developed in the epiphyseal plate [12].

Soeta et al. investigated the growth plate cartilage immunohistochemically in proximal tibia in rats administered high-dose vitamin A. From the $1^{\text {st }}$ to $5^{\text {th }}$ day, they assessed changes using antibodies against type I, type II and type $X$ collagen. On the $5^{\text {th }}$ day type I and type II collagen were partly lost, while positive type $X$ collagen areas reduced on the $4^{\text {th }}$ day and were clearly lost by the $5^{\text {th }}$ day [11]. In our study, immunohistochemical studies at the end of the $4^{\text {th }}$ week did not encounter type II collagen in the epiphyseal bone, proliferative zone and hypertrophic zone in the control group, low-dose acitretin solution group and the high-dose acitretin solution group. Type II collagen was not observed in osteoids and osteoblasts. Type I collagen was not observed in the hypertrophic zone and proliferative zone of all groups. In all groups the intensity of type I collagen in the epiphyseal bone, osteoids and osteoblasts was identified as $4(+++)$. There were no significant differences identified in terms of Type I collagen staining distribution and intensity between the groups. Again, in this study by Soeta et al. columnar arrangement was observed in the control group but the columnar arrangement of chondrocytes was lost after 3 days in rats administered vitamin A. The thickness of the epiphyseal plate was 40\% reduced on the $5^{\text {th }}$ day compared to the control group [11]. In this study, the columnar arrangement of chondrocytes in the epiphyseal growth plate was $90.9 \%$ in the control group, 9.1\% in the low-dose acitretin group and clearly reduced and lost in the high-dose acitretin group. According to these data, we showed the columnar arrangement was lost with an increasing acitretin dose. In our study, though there was significant thinning of the hypertrophic zone of the epiphyseal plate, there was no significant reduction in plate thickness. We concluded that acitretin did not cause a clear reduction in growth plate height.

Another study by Soeta et al. investigated the focal loss of epiphyseal growth plate cartilage in rats administered high-dose vitamin A. The epiphyseal plate was assessed 1 week and 2 weeks (12 ${ }^{\text {th }}$ and $19^{\text {th }}$ days) after 5 days of vitamin A administration. On the $12^{\text {th }}$ day, it appeared that abnormal cartilage matrix had developed in the epiphyseal growth plate of rats administered vitamin A. On the $19^{\text {th }}$ day there was a reduction in columnar arrangement and one or two focal losses in the epiphyseal growth plate of all rats administered vitamin A. Additionally, the calcified cartilage matrix surfaces were covered with a fine layer of bone tissue [13].

Francesco de Luca et al. cultivated foetal rat metatarsal bones in the presence of retinoic acid to determine whether there was a direct effect of retinoic acid on the growth plate. Single-dose oral retinoic acid treatment was shown to reduce the height of the proximal tibial growth plate in rats [16].

An animal study by Descalzi Cancedda et al. showed that retinoic acids reduced type II collagen synthesis and increased type I collagen synthesis. Studies proposed that the increase in type I collagen was due to chondrocytes transforming into an osteoblast-like phase [17].

Some studies have reported that excess vitamin A increases the number and volume of osteoclasts, reduces the osteoid surface causing bone resorption and leads to osteoporosis and bone fractures. Human studies with synthetic retinoids have shown retinoids increase bone metabolism and turnover [5].

In spite of different results from human studies, current literature analysis shows that higher or lower serum retinol levels compared to normal or diets high in vitamin A cause an increase in the risk of hip fractures [6].

The literature reports a case with broad hyperostosis development causing significant disability after 13 years of retinoid use $[18,19]$.

A broad study of 240 patients treated with acitretin for 6 months found that only $5 \%$ of the patients developed bone abnormalities. However, it is not clear whether this situation is linked to the medication or the normal process. There is no relationship described between the dose or treatment duration with bone abnormalities [4].

A patient using acitretin for 20 years due to lamellar ichthyosis observed clear resorption of the terminal phalanx of the left index finger and found initial findings of resorption in the other fingers. 


\section{Conclusions}

Though this study of acitretin found variations in the epiphyseal growth plate and bone metabolism, we identified no significant reduction in the epiphyseal plate or bone demineralization.

\section{Acknowledgments}

We thank the Yuzuncu Yıl University Scientific Research Project Chair for funding this research.

This study was conducted in the Department of Dermatology of the Yuzuncu Yıl University.

\section{Conflict of interest}

The authors declare no conflict of interest.

\section{References}

1. Ellis CN, Krach KJ. Uses and complications of isotretinoin therapy. J Am Acad Dermatol 2001; 45: S150-7.

2. Luthi F, Eggel Y, Theumann N. Premature epiphyseal closure in an adolescent treated by retinoids for acne: an unusual cause of anterior knee pain. Joint Bone Spine 2012; 79: 314-6.

3. Nilsson O, Isoherranen N, Guo MH, et al. Accelerated skeletal maturation in disorders of retinoic acid metabolism: a case report and focused review of the literature. Horm Metab Res 2016; 48: 737-44.

4. Halverstam CP, Zeichner J, Lebwohl M. Lack of significant skeletal changes after long-term, low-dose retinoid therapy: case report and review of theliterature. J Cutan Med Surg 2006; 10: 291-9.

5. Penniston KL, Tanumihardjo SA. The acute and chronic toxic effects of vitamin A. Am J Clin Nutr 2006; 83: 191-201.

6. Green AC, Kocovski P, Jovic T, et al. Retinoic acid receptor signalling directly regulates osteoblast and adipocyte differentiation from mesenchymal progenitor cells. Exp Cell Res 2017; 350: 284-97.

7. Koyama E, Golden EB, Kirsch T, et al. Retinoid signaling is required for chondrocyte maturation and endochondral bone formation during limb skeletogenesis. Dev Biol 1999; 208: 375-91.

8. Burdan F, Szumito J, Korobowicz A, et al. Morphology and physiology of theepiphyseal growth plate. Folia Histochem Cytobiol 2009; 47: 5-16.

9. Shim KS. Pubertal growth and epiphyseal fusion. Ann Pediatr Endocrinol Metab 2015; 20: 8-12.

10. Ballock RT, O'Keefe RJ. Physiology and pathophysiology of the growth plate. Birth Defects Res C Embryo Today 2003; 69: $123-43$

11. Soeta S, Mori R, Kodaka T, et al. Immunohistochemical observations on the initial disorders of the epiphyseal growth plate in rats induced by high dose of vitamin A. J Vet Med Sci 1999; 61: 233-8.

12. Kodaka T, Takaki H, Soeta S, et al. Local disappearance of epiphyseal growth plates in rats with hypervitaminosis A. J Vet Med Sci 1998; 60: 815-21.

13. Soeta S, Mori R, Kodaka T, et al. Histological disorders related to the focal disappearance of the epiphyseal growth plate in rats induced by high dose of vitamin A. J Vet Med Sci 2000; 62: 293-9.
14. Yıldızgören MT, Karataş Toğral A, Baki AE, et al. Effects of isotretinoin treatment on cartilage and tendon thicknesses: an ultrasonographic study. Clin Rheumatol 2015; 34: 1255-8.

15. DiGiovanna JJ. Isotretinoin effects on bone. J Am Acad Dermatol 2001; 45: S176-82.

16. De Luca F, Uyeda JA, Mericq V, et al. Retinoic acid is a potent regulator of growth plate chondrogenesis. Endocrinology 2000; 141: 346-53.

17. Descalzi Cancedda F, Gentili C, Manduca P, Cancedda R. Hypertrophic chondrocytes undergo further differentiation in culture. J Cell Biol 1992; 117: 427-35.

18. van Dooren-Greebe RJ, van de Kerkhof PC. Extensive extraspinal hyperostoses after long-term oral retinoid treatment in a patient with pityriasis rubra pilaris. J Am Acad Dermatol 1995; 32: 322-5.

19. Saravana S, Wittkop B, Rai A. Distal terminal phalanx bone resorption and long-term acitretin therapy. Int I Dermatol 2008; 47: 1211-2. 